Research Article

\title{
Performance Evaluation of MU-MIMO Transmissions with Joint Interference Constraint in HetNet
}

\author{
Wenjian Zhang (iD) and Senlin Jiang (iD) \\ Wuxi Institute of Technology, Wuxi, China \\ Correspondence should be addressed to Wenjian Zhang; zhangwj@wxit.edu.cn
}

Received 9 October 2021; Accepted 2 November 2021; Published 23 November 2021

Academic Editor: Zhihan Lv

Copyright (c) 2021 Wenjian Zhang and Senlin Jiang. This is an open access article distributed under the Creative Commons Attribution License, which permits unrestricted use, distribution, and reproduction in any medium, provided the original work is properly cited.

In this paper, we investigate the per-tier outage probability of multiuser multiple-input multiple-output (MU-MIMO) transmissions in heterogeneous networks (HetNets) with joint interference constraint. In the tier of cellular cell, user equipment (UE) is required to report measured channel information and the base station (BS) adopts ZF-based precoding MU-MIMO transmission to achieve multiuser diversity gain. With the constraint of cross-tier interference and unpredicted inter-beam interference, we derive the closed-form expression of outage probability of downlink MU-MIMO transmissions. Considering the capacity of nodes in the tier of ad hoc networks, a max-SINR scheduler and codebook-based MU-MIMO transmission are employed. The scheduler selects the best receiving nodes for each beam in predefined codebook according to measured signal to interference plus noise ratio (SINR), and the transmitting node performs data transmissions using orthogonal beams. In the presence of inter-node interference, inter-beam interference, and cross-tier interference, we obtain the closed-form expression of outage probability of MU-MIMO transmissions when downlink or uplink transmissions occur in cellular cell. Additionally, in case that the outage probability in ad hoc networks should satisfy quality of service (QoS) requirement, a restricted area in cellular cell in which the outage probability in ad hoc networks is not greater than a required threshold is explored. Numerical results show that the unpredictable inter-beam interference in cellular cell degrades the outage probability slightly. The restricted area increases with the outage probability threshold.

\section{Introduction}

Wireless ad hoc network is an autonomous network consisting of a collection of peer nodes equipped with wireless transceiver. The nodes communicate with each other directly or by multihop link with the help of intermediate nodes. Due to the flexible structure, ad hoc networks have attracted a lot of attention in different application areas, such as military battlefield [1], emergency mission, postdisaster reconstruction [2], and vehicular communication [3]. In this paper, the temporary ad hoc networks without infrastructure and the widely deployed cellular cells constitute HetNets [4], as shown in Figure 1.

Due to the scarcity of frequency resource and lack of coordination, the HetNets usually share the same channels. The occurrence of cross-tier interference with each other is inevitable. In order to lower the impact on the performance of the coexisting system, some interference coordination schemes, such as spectrum allocation, power control, and interference cancellation, have been studied in prior works [5-7]. Since there are several transmitting nodes in the ad hoc networks that send data on the same channels simultaneously, a receiving node experiences the inter-node interference signal from other transmitting nodes additionally. The effect of inter-node interference signal was investigated in $[8]$.

With the goal of improving the performance of HetNets, MU-MIMO technology has been employed. Due to its extremely high spectral efficiency, MU-MIMO has received a significant attention [9-11]. In the 3rd Generation Partnership Project (3GPP) Long-Term Evolution-Advanced (LTE-A) [12] and IEEE 802.11ac [13], the MU-MIMO has 


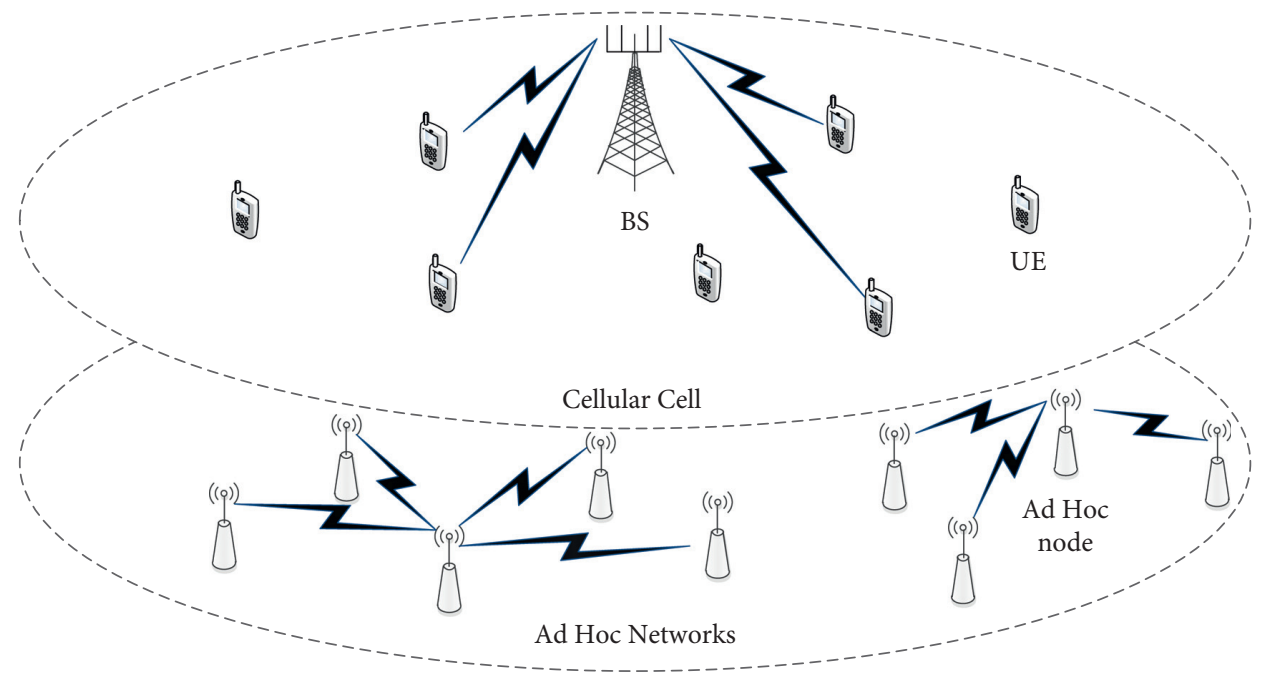

Figure 1: HetNet consisting of cellular cell and ad hoc network with MU-MIMO transmissions.

been adopted. In MU-MIMO transmissions, according to the measured information feedback from receivers, the forward transmission could be achieved in different ways. In the first way, the receivers perform channel information measurement and feed back the measured information to the transmitter. The scheduler selects the matching receivers and assigns orthogonal beams. Then, the transmitter sends data to the selected receivers using the assigned beams. The precoding matrixes of beamforms are calculated according to the feedback channel information by some algorithms, such as SVD [14], block diagonalization [15], zero-forcing (ZF) precoding, and dirty-paper coding [16]. In the second way, the precoding matrixes are predefined in codebook. The receivers perform the SINR measurement for each predefined beam and report the measured SINRs to the transmitter. The max-SINR scheduler selects the best receivers for each beam according to the reported SINRs [17]. Then, the transmitter simultaneously transmits data to the selected receivers, using orthogonal random beams.

The first way is applicable to the situation that the transmitter could get the full channel information. In case of perfect channel state information (CSI), the beams for each receiver keep orthogonal in data transmission phase. If perfect CSI could not be obtained due to some reasons, such as quantization error, the beams interfere with each other. In the second way, since the max-SINR scheduler does not need perfect CSI, the beam for a selected receiver may mismatch with its forward channel in data transmission phase. Hence, the beams for each receiver interfere with each other. The inter-beam interference impairs the performance of MUMIMO transmissions inevitably.

Moreover, the cross-tier interference signal that a receiving node suffers may come from the cellular BS in a downlink interval. Because the transmitting power of BS is much greater than that of a transmitting node, the receiving nodes close to the BS may experience a high outage probability which could not satisfy the QoS requirements. This means that there is a performance restricted area around the BS in which the outage probability of a receiving node exceeds a required threshold. The area of the restricted area is worth exploring.

Motivated by the discussion above, in the paper, we evaluate per-tier outage probability performance of MUMIMO transmissions with joint interference constraint in HetNets. Our main contributions are given as follows:

(i) We adopt different MU-MIMO transmission models in cellular cell and ad hoc networks according to the capacity of equipment and the complexity of network structure. The models have different complexities and transmission performances.

(ii) We derive per-tier closed-form outage probability of MU-MIMO transmissions with the joint interference constraint.

(iii) We explore the restricted area in cellular cell in which the outage probability of the MU-MIMO transmissions in ad hoc networks could not satisfy QoS requirements.

The remainder of the paper is structured as follows. Section 2 gives network model and transmission model. Section 3 derives per-tier outage probability of MU-MIMO transmissions in the presence of joint interference. Section 4 explores the restricted area in cellular cell. Section 5 describes the numerical results. Finally, Section 6 gives the conclusions.

\section{System Model}

We assume a HetNet consisting of a time division multiplexing access (TDMA) cellular system and coexisting ad hoc networks in this paper.

2.1. Network Model. In cellular cell, the cellular BS $B_{0}$ at the center serves a circle area. The UEs are distributed in the coverage of $B_{0}$ according to a spatial Poisson point process (SPPP) with intensity $\lambda_{c}$. Hence, the UEs are denoted as 
$\Psi\left(\lambda_{c}\right)=\left\{Z_{\psi}\right\}$, where $Z_{\psi}$ is the position of UE $\psi \in Z_{\psi}$. The model has been used, and the validity has been confirmed in prior works [18-20].

In coexisting ad hoc networks, we assume that the ALOHA slotted protocol is adopted [21]. In each slot, a subset of nodes transmits data on the same channel simultaneously. The transmitting nodes are assumed to be distributed according to a SPPP with intensity $\lambda_{f}$. Similarly, the transmitting nodes are denoted as $\Phi\left(\lambda_{f}\right)=\left\{Z_{\varphi}\right\}$.

2.2. Transmission Model. The BS $B_{0}$ and the transmitting nodes in ad hoc networks are assumed to be equipped with $N_{c}$ and $N_{f}$ transmitting antennas, respectively, and the receivers are assumed to be equipped with single receiving antenna.

In cellular cell, the CSI feedback is widely employed, as in the LTE system. It is easy for the BS to obtain the feedback CSI from UE, and thus the linear ZF-based precoding transmission is assumed to be applied in downlink interval due to its low complexity. With ZF-based precoding transmission, the $\mathrm{BS} B_{0}$ chooses its precoding matrix $\mathbf{W} \in \mathbb{C}^{N_{c} \times K_{c}}=\left[\mathbf{w}_{i}\right]_{1 \leq i \leq K_{c}}$ as the normalized columns of the pseudo-inverse $\mathbf{H}^{*}\left(\mathbf{H} \mathbf{H}^{*}\right)^{-1} \in \mathbb{C}^{N_{c} \times K_{c}}$, where $\mathbf{H}^{*}$ represents the conjugate transpose of $\mathbf{H}$ and $K_{c}\left(1 \leq K_{c} \leq N_{c}\right)$ is number of selected UEs. In the data transmission phase, the BS transmits data to the selected UE using the beamsw $_{i}\left(i=1,2, \ldots, K_{c}\right)$. The total power of BS is assumed to be $P_{c}$. In uplink interval of cellular cell, all scheduled UEs send data packet to the BS simultaneously with the same power $P_{m}$

Considering that the processing capacity of ad hoc node is weaker than that of cellular BS, the real-time calculation of precoding matrixes could not be achieved. Hence, the codebook-based MU-MIMO transmission with finite-rate feedback is adopted in ad hoc networks. In each slot, the max-SINR scheduler of transmitting node selects $K_{f}\left(1 \leq K_{f} \leq N_{f}\right)$ receiving nodes for each beam based on measured SINRs. In the data transmission phase, the transmitting node transmits data to the selected receiving nodes simultaneously, using random orthogonal beams $\mathbf{v}_{k}\left(k=1,2, \ldots, K_{f}\right)$ [17]. The total power of a transmitting node is assumed to be $P_{f}$.

\section{Per-Tier Outage Probability Evaluation}

In this section, we evaluate per-tier outage probability of MU-MIMO transmissions with joint interference constraint and give the closed-form expressions of outage probability.

3.1. Outage Probability of Downlink MU-MIMO Transmissions in Cellular Cell. We assume that UE $i$ is located at the origin. In downlink interval, the UE $i$ suffers the unpredicted interbeam interference signal and cross-tier interference signal. Hence, the instantaneous received signal for the UE $i$ is denoted as

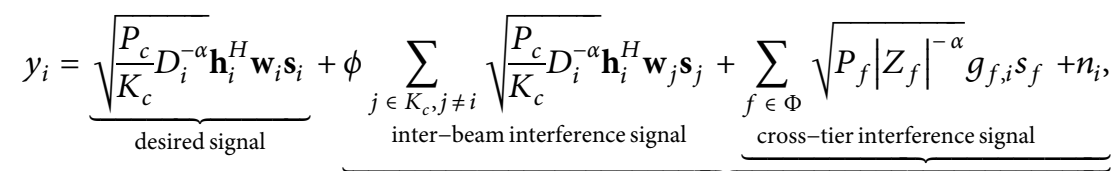

where $\mathbf{h}_{i} \in \mathbb{C}^{N_{c} \times 1}$ is the channel gain vector between UE $i$ and the BS $B_{0}$, whose entries follow complex Gaussian distribution $h_{i, j} \sim \mathrm{CN}(0,1)$. Similarly, $g_{f, i}$ is the channel gain between UE $i$ and a transmitting node in ad hoc networks. $\mathbf{s}_{i}$ is the symbol vector for UE $i . n_{i}$ represents the additive white Gaussian noise (AWGN) with zero mean and variance of $\sigma^{2}$. $\phi$ is a switching variable. In case of perfect CSI or $K_{c}=1, \phi=0$. Otherwise, $\phi=1$.

It is obvious that the system is an interference-limited system. Hence, the effect of thermal noise could be ignored for analysis simplicity. The instantaneous received SINR is denoted as

$$
\begin{aligned}
\gamma_{i} & =\frac{P_{c} D_{i}^{-\alpha} / K_{c}\left|\mathbf{h}_{i}^{H} \mathbf{w}_{i}\right|^{2}}{\phi\left(P_{c} D_{i}^{-\alpha} / K_{c}\right) \sum_{j \in K_{c}, j \neq i}\left|\mathbf{h}_{i}^{H} \mathbf{w}_{j}\right|^{2}+P_{f} \sum_{f \in \Phi}\left|Z_{f}\right|^{-\alpha}\left|g_{f, i}\right|^{2}} \\
& =\frac{\vartheta S}{\phi \vartheta I_{\mathrm{IBI}}+I_{\mathrm{CTI}}},
\end{aligned}
$$

where $\vartheta=P_{c} D_{i}^{-\alpha} / K_{c}$. The random variables $S=\left|\mathbf{h}_{i}^{H} \mathbf{w}_{i}\right|^{2}$ and $I_{\mathrm{IBI}}=\sum_{j \in K_{c}, j \neq i}\left|\mathbf{h}_{i}^{H} \mathbf{w}_{j}\right|^{2}$ are chi-square random variables with $2\left(N_{c}-K_{c}+1\right)$ and $2\left(K_{c}-1\right)$ degrees of freedom, respectively. The random variable $I_{\mathrm{CTI}}=P_{f} \sum_{f \in \Phi}\left|Z_{f}\right|^{-\alpha}\left|g_{f, k}\right|^{2}$ represents the power of crosstier interference signal.

The outage probability of an MU-MIMO transmission is defined as the probability that instantaneous received SINR does not exceed the required SINR threshold. Assuming that

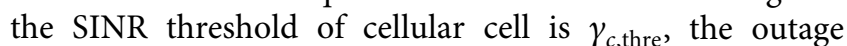
probability of downlink MU-MIMO transmissions is denoted as

$$
\begin{aligned}
p_{i}\left(D_{i}\right) & =\mathbb{P}\left(\gamma_{i} \leq \gamma_{c, \text { thre }}\right) \\
& =\mathbb{P}\left(S \leq \omega\left(\phi \vartheta I_{\mathrm{IBI}}+I_{\mathrm{CTI}}\right)\right) \\
& =\int_{0}^{\infty} \mathbb{P}(S \leq \omega x) f_{\phi 9 I_{\mathrm{IBI}}+I_{\mathrm{CTI}}}(x) \mathrm{d} x,
\end{aligned}
$$

where $\omega=\gamma_{c \text {,thre }} / \vartheta$. 
Note that the random variable $S$ obeys chi-square distribution with $2\left(N_{c}-K_{c}+1\right)$ degrees of freedom. Hence, the term $\mathbb{P}(S \leq \omega x)$ is denoted as

$$
\begin{aligned}
\mathbb{P}(S \leq \omega x) & =\int_{0}^{\omega x} \frac{t^{N_{c}-K_{c}}}{2^{N_{c}-K_{c}+1}\left(N_{c}-K_{c}\right) !} e^{-(t / 2)} \mathrm{d} t \\
& =1-\frac{1}{\left(N_{c}-K_{c}\right) !} \Gamma\left(N_{c}-K_{c}+1, \omega x\right) \\
& =1-e^{-\omega x} \sum_{m=0}^{N_{c}-K_{c}} \frac{(\omega x)^{m}}{m !}
\end{aligned}
$$

$$
p_{i}\left(D_{i}\right)=\left\{1-\sum_{m=0}^{N_{c}-K_{c}} \frac{(-\omega)^{m}}{m !} \frac{d^{m}}{d \omega^{m}} \mathscr{L}_{I_{\mathrm{CTI}}}(\omega), \phi=0,1-\sum_{m=0}^{N_{c}-K_{c}} \frac{(-\omega)^{m}}{m !} \frac{d^{m}}{d \omega^{m}} \mathscr{L}_{I_{\mathrm{IBI}}}(\vartheta \omega) \mathscr{L}_{I_{\mathrm{CTI}}}(\omega), \phi=1,\right.
$$

where $\mathscr{L}_{V}(\cdot)$ denotes the Laplace transform of the random variable $V$. The transform of $\mathscr{L}\left[t^{m} f(t)\right]=(-1)^{m} F^{(m)}(s)$ is employed.

Since $I_{\mathrm{IBI}}$ is the chi-square random variable with $2\left(K_{c}-\right.$ 1) degrees of freedom, the Laplace transform of $I_{\mathrm{IBI}}$ and its $m$-th order differential are, respectively, defined as

$$
\begin{aligned}
\mathscr{L}_{I_{\mathrm{IBI}}}(\vartheta \omega) & =\frac{1}{\left(1+\gamma_{c, \text { thre }}\right)^{K_{c}-1}} \\
\frac{d^{m}}{d \omega^{m}} \mathscr{L}_{I_{\mathrm{IBI}}}(\vartheta \omega) & =\frac{(-\vartheta)^{m}}{(1+\vartheta \omega)^{K_{c}+m-1}} \prod_{j=0}^{m-1}\left(K_{c}+j-1\right) .
\end{aligned}
$$

In addition, the transmitting nodes in a slot are distributed according to a SPPP with intensity $\lambda_{f}$, and hence the cross-tier interference signal is modeled as a general Poisson shot noise process [22]. The Laplace transform of $I_{\mathrm{CTI}}$ could be obtained as

$$
\begin{aligned}
\mathscr{L}_{I_{\mathrm{CTI}}}(\omega) & =\exp \left\{-\lambda_{f} \int_{\mathbb{R}^{2}} 1-\mathbb{E}_{H}\left[\exp \left(-\omega H|x|^{-\alpha}\right)\right]\right\} \\
& =\exp \left\{-2 \pi \lambda_{f} \int_{0}^{\infty} \frac{x}{1+|x|^{\alpha} / \omega} \mathrm{d} x\right\} \\
& =\exp \left(-\lambda_{f} \mathrm{Q} \omega^{\delta}\right)
\end{aligned}
$$

where $\Gamma(a, t)=\int_{0}^{t} x^{a-1} e^{-x} \mathrm{~d} x$ is complementary incomplete gamma function with $a>0$. Since the variables $I_{\mathrm{IBI}}$ and $I_{\mathrm{CTI}}$ are independent random variables, substituting the result in equation (4) into (3), the outage probability of downlink MU-MIMO transmissions is rewritten as where $\delta=2 / \alpha$ and $Q=\pi P_{f}^{\delta} \Gamma(\delta) \Gamma(1-\delta)$.

If a high probability of successful reception in cellular cell is required, it is reasonable that the density of transmitting nodes $\lambda_{f}$ is very small. Hence, the $m$-th order differential of $\mathscr{L}_{I_{\mathrm{CTI}}}(\omega)$ can be evaluated using first-order Taylor series around $\lambda_{f} Q \omega^{\delta}=0$. For all $m \geq 1$, the $m$-th order differential of $\mathscr{L}_{I_{\mathrm{CTI}}}(\omega)$ is given approximately as

$$
\begin{aligned}
& \frac{d^{m}}{d \omega^{m}} \mathscr{L}_{I_{\mathrm{CTI}}}(\omega) \\
& \approx-\left(\lambda_{f} \mathrm{Q} \omega^{\delta-m} \prod_{l=0}^{m-1}(\delta-m)\right) e^{-\lambda_{f} \mathrm{Q} \omega^{\delta}}+\Psi\left(\lambda_{f}^{2} Q^{2} \omega^{2 \delta}\right) .
\end{aligned}
$$

In case of $\phi=0$, the outage probability of downlink MUMIMO transmissions is denoted as

$$
p_{i}\left(D_{i}\right)= \begin{cases}1-\exp \left(-\lambda_{f} Q \omega^{\delta}\right), & K_{c}=N_{c}, \\ \lambda_{f} Q \omega^{\delta}\left(1+\sum_{m=1}^{N_{c}-K_{c}} \frac{1}{m !} \prod_{l=0}^{m-1}(1-\delta)\right), 1 \leq K_{c}<N_{c} .\end{cases}
$$

In case of $\phi=1$ and $K_{c}=N_{c}$, the outage probability is denoted as

$$
p_{i}\left(D_{i}\right)=1-\frac{e^{-\lambda_{f} \mathrm{Q} \omega^{\delta}}}{\left(1+\gamma_{c, \text { thre }}\right)^{K_{c}-1}}
$$


If $1<K_{c}<N_{c}$, the outage probability is denoted as

$$
\begin{aligned}
p_{i}\left(D_{i}\right)= & 1-\sum_{m=0}^{N_{c}-K_{c}} \frac{(-\omega)^{m}}{m !} \sum_{l=0}^{m}\left(\begin{array}{c}
m \\
l
\end{array}\right) \frac{d^{l}}{d \omega^{l}} \mathscr{L}_{I_{\mathrm{IBI}}}(\omega) \frac{d^{m-l}}{d \omega^{m-l}} \mathscr{L}_{I_{\mathrm{CTI}}}(\omega) \\
= & \frac{\mathscr{F} \frac{\gamma_{c, \text { thre }}}{1+\gamma_{c, \text { thre }}}\left(N_{c}-K_{c}+1, K_{c}-1\right)}{} \\
& +\lambda_{f} Q \omega^{\delta}\left(\mathscr{K}\left(K_{c}-1\right)-\mathscr{F} \frac{\gamma_{c, \text { thre }}}{1+\gamma_{c, \text { thre }}}\left(N_{c}-K_{c}+1, K_{c}-1\right)\right) .
\end{aligned}
$$

For the definitions of the function $\mathscr{F}_{x}(a, b)$ and the variable $\mathscr{K}(a)$, refer to the work [9].

3.2. Outage Probability of MU-MIMO Transmissions in Ad Hoc Network. Firstly, it is noted that some intermediate variables in the previous section are reused in this section for expression simplicity.

In ad hoc networks, a receiving node suffers inter-node interference signal additionally. Hence, for a receiving node $k$ located at the origin, the received signal for the assigned beam $\mathbf{v}_{k}$ is denoted as

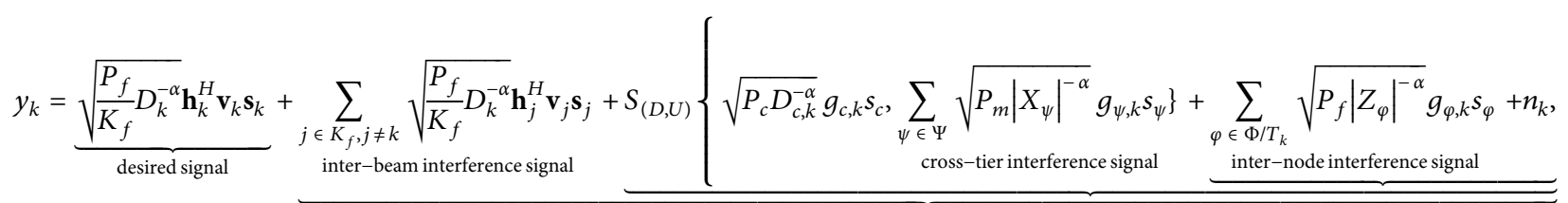

where $\mathbf{h}_{k} \in \mathbb{C}^{N_{f} \times 1}$ is the channel gain vector between the receiving node $k$ and the transmitting node. $g_{\varphi, k}$ is the channel gain between receiving node $k$ and a transmitter $\varphi$, which follows Rayleigh distribution. $S_{(D, U)}\left\{S_{1}, S_{2}\right\}$ represents $S=S_{1}$ in case of $D$ or $S=S_{2}$ in case of $U$. Hence, the crosstier interference signal from cellular cell is $\sqrt{P_{c} D_{c, k}^{-\alpha}} g_{c, k} s_{c}$ in case of downlink interval or $\sum_{\psi \in \Psi} \sqrt{P_{m}\left|X_{\psi}\right|^{-\alpha}} g_{\psi, k} s_{\psi}$ in case of uplink interval.

Note that the cross-tier interference signal in the data transmission phase may change to be different from that of
SINR measurement. If the power of instantaneous cross-tier interference signal increases, the receiving node that has the best SINR for the assigned beam may be changed. However, the receiving node could not be adjusted during data transmission. The performance of MU-MIMO transmissions may degrade.

For the receiving node $k$, the instantaneous received SINR for the assigned beam $\mathbf{v}_{k}$ is denoted as

$$
\begin{aligned}
\gamma_{k} & =\frac{\left(P_{f} / K_{f}\right) D_{k}^{-\alpha}\left|\mathbf{h}_{k}^{H} \mathbf{v}_{k}\right|^{2}}{\phi\left(P_{f} D_{k}^{-\alpha} / K_{f}\right) \sum_{j \in K_{f}, j \neq k}\left|\mathbf{h}_{j}^{H} \mathbf{v}_{j}\right|^{2}+P_{f} \sum_{\varphi \in \Phi / T_{k}}\left|Z_{\varphi}\right|^{-\alpha}\left|g_{\varphi, k}\right|^{2}+I_{\mathrm{CTI}}} \\
& =\frac{\vartheta S}{\phi \vartheta I_{\mathrm{IBI}}+I_{\mathrm{INI}}+I_{\mathrm{CTI}}},
\end{aligned}
$$

where $\vartheta=P_{f} D_{k}^{-\alpha} / K_{f}$. The definition of random variables $S$ and $I_{\mathrm{IBI}}$ is similar to that in equation (2). In terms of the properties of the SPPP model, the random variable $I_{\text {INI }}$ has same property as the variable $I_{\text {CTI }}$ in equation (2). The random variable $I_{\mathrm{CTI}}$ is dependent on the type of interval in cellular cell. In case of downlink interval, 
$I_{\mathrm{CTI}}=P_{c}\left|D_{c, k}\right|^{-\alpha}\left|g_{c, k}\right|^{2}$. In case of uplink interval, $I_{\text {CTI }}=\sum_{\psi \in \Psi} P_{m}\left|X_{\psi}\right|^{-\alpha}\left|g_{\psi, k}\right|^{2} . \phi$ is a switching variable. In case $K_{f}=1, \phi=0$. Otherwise, $\phi=1$.

Similarly, we assume that the SINR threshold in ad hoc networks is $\gamma_{f \text {,thre }}$. Referring to equation (3), the outage probability is denoted as

$$
p_{k}\left(D_{k}\right)=\mathbb{P}\left(S \leq \omega\left(\phi \vartheta I_{\mathrm{IBI}}+I_{\mathrm{INI}}+I_{\mathrm{CTI}}\right)\right),
$$

where $\omega=\gamma_{f \text {,thre }} / \mathcal{\vartheta}$.

Considering that the random variable $S$ obeys exponential distribution and $I_{\mathrm{IBI}}, I_{\mathrm{INI}}$, and $I_{\mathrm{CTI}}$ are independent random variables, the outage probability is expressed as

$$
p_{k}\left(D_{k}\right)= \begin{cases}1-\mathscr{L}_{I_{\mathrm{INI}}}(\omega) \mathscr{L}_{I_{\mathrm{CTI}}}(\omega), & \phi=0, \\ 1-\mathscr{L}_{I_{\mathrm{IBI}}}(\vartheta \omega) \mathscr{L}_{I_{\mathrm{INI}}}(\omega) \mathscr{L}_{I_{\mathrm{CTI}}}(\omega), & \phi=1 .\end{cases}
$$

Since $I_{\mathrm{IBI}}$ is the chi-square random variable with $2\left(K_{f}-\right.$ 1) degrees of freedom, the Laplace transform of $I_{\mathrm{IBI}}$ is defined as

$$
\mathscr{L}_{I_{\mathrm{IBI}}}(\vartheta \omega)=\frac{1}{(1+\vartheta \omega)^{K_{f}-1}} .
$$

The inter-node interference signal could be modeled as a general Poisson shot noise process. Hence, the Laplace transform of $I_{\text {INI }}$ is the same as the result in equation (7), i.e.,

$$
\mathscr{L}_{I_{\mathrm{INI}}}(\omega)=\exp \left(-\pi \lambda_{f} B(\delta, 1-\delta)\left(\omega P_{f}\right)^{\delta}\right)
$$

where $B(a, b)=\Gamma(a) \Gamma(b) / \Gamma(a+b)$ denotes the beta function with gamma function $\Gamma(a)=\int_{0}^{\infty} x^{a-1} e^{-x} \mathrm{~d} x$.

If the cross-tier interference signal comes from UEs in uplink interval, since the UEs are distributed according to a SPPP with intensity $\lambda_{c}$, similarly the term of $\mathscr{L}_{I_{\text {CTI }}}(\omega)$ is given as

$$
\mathscr{L}_{I_{\mathrm{CTI}}}(\omega)=\exp \left(-\pi \lambda_{c} B(\delta, 1-\delta)\left(\omega P_{m}\right)^{\delta}\right) .
$$

Substituting the results in equations (16) (18) into equation (15), the outage probability of MU-MIMO transmissions is denoted as

$$
p_{k}\left(D_{k}\right)= \begin{cases}1-e^{-\pi \omega^{\delta}\left(\lambda_{f} P_{f}^{\delta}+\lambda_{c} P_{m}^{\delta}\right) B(\delta, 1-\delta)}, & \phi=0, \\ 1-\frac{e^{-\pi \omega^{\delta}\left(\lambda_{f} P_{f}^{\delta}+\lambda_{c} P_{m}^{\delta}\right) B(\delta, 1-\delta)}}{\left(1+\gamma_{f, \text { thre }}\right)^{K_{f}-1}}, & \phi=1 .\end{cases}
$$

If the cross-tier interference signal comes from cellular BS in downlink interval, taking the Rayleigh fading channel into account, the term of $\mathscr{L}_{I_{\mathrm{CTI}}}(\omega)$ is given as

$$
\mathscr{L}_{I_{\mathrm{CTI}}}(\omega)=\frac{1}{1+\omega P_{c} D_{c, k}^{-\alpha}} .
$$

By using the results in equations (16) (17) and (20), the outage probability is denoted as

$$
p_{k}\left(D_{k}\right)= \begin{cases}1-\frac{e^{-\pi \lambda_{f}\left(\omega P_{f}\right)^{\delta} B(\delta, 1-\delta)}}{\left(1+\omega P_{c} D_{c, k}^{-\alpha}\right)}, & \phi=0, \\ 1-\frac{e^{-\pi \lambda_{f}\left(\omega P_{f}\right)^{\delta} B(\delta, 1-\delta)}}{\left(1+\omega P_{c} D_{c, k}^{-\alpha}\right)\left(1+\gamma_{f, \text { thre }}\right)^{K_{f}-1}}, & \phi=1 .\end{cases}
$$

\section{Restricted Area in Cellular Cell}

For a receiving node in MU-MIMO transmission in ad hoc networks, it suffers the cross-tier interference signal from cellular cell. Considering that the transmitting power of cellular BS is greater than that of a transmitting node, the cross-tier interference signal may severely impair the performance of MU-MIMO transmissions in ad hoc networks during the downlink interval.

For the receiving nodes close to the cellular BS, their outage probability performance may not satisfy the QoS requirements. Hence, the instantaneous outage probability is not greater than a required threshold, i.e.,

$$
p_{k}\left(D_{k}\right)=1-\frac{P_{c} D_{c, k}^{-\alpha} e^{-\pi \omega^{\delta}}\left(\lambda_{f} P_{f}^{\delta}\right) B(\delta, 1-\delta)}{(1+\omega)(1+\vartheta \omega)^{K_{f}-1}} \leq \varepsilon,
$$

where $\varepsilon$ is the outage probability threshold. Consequently, the distance between BS $B_{0}$ and the receiving node $k$ should satisfy

$$
\begin{aligned}
D_{c, k} & \geq\left(\frac{P_{c} e^{-\pi \omega^{\delta}\left(\lambda_{f} P_{f}^{\delta}\right) B(\delta, 1-\delta)}}{(1-\varepsilon)(1+\omega)\left(1+\gamma_{f, \text { thre }}\right)^{K_{f}-1}}\right)^{1 / \alpha} \\
& >\left(\frac{P_{c}\left(1-\pi \omega^{\delta}\left(\lambda_{f} P_{f}^{\delta}\right) B(\delta, 1-\delta)\right)}{(1-\varepsilon)(1+\omega)\left(1+\gamma_{f, \text { thre }}\right)^{K_{f}-1}}\right)^{1 / \alpha} .
\end{aligned}
$$

Considering $\omega=K_{f} \gamma_{f \text {,thre }} /\left(P_{f} D_{k}^{-\alpha}\right)$, the distance $D_{c, k}$ is the subtractive function of the distance $D_{k}$. Intuitively, the decrease of the distance $D_{k}$ improves the power of desired signal at a receiving node, and thus more receiving nodes could tolerate the cross-tier interference to satisfy QoS requirements.

Furthermore, we assume that the minimum distance between a transmitting node and its receiving node is $R$, and hence the minimum value of $D_{c, k}$ is denoted as

$$
D_{c, k}^{\min }=\left(\frac{P_{c} P_{f}\left(1-\pi \lambda_{f} R^{2}\left(K_{f} \gamma_{f, \text { thre }}\right)^{\delta} B(\delta, 1-\delta)\right)}{(1-\varepsilon)\left(P_{f}+K_{f} R^{\alpha} \gamma_{f, \text { thre }}\right)\left(1+\gamma_{f, \text { thre }}\right)^{K_{f}-1}}\right)^{1 / \alpha} .
$$


TABLE 1: Simulation configurations.

\begin{tabular}{lc}
\hline Parameter & Value \\
\hline Total transmitting power $P_{c} / P_{f} / P_{m}$ & $46 / 23 / 13 \mathrm{dBm}$ \\
Transmitting antennas $N_{c}$ and $N_{f}$ & 8 \\
Receiving UE $K_{c}$ & 4,8 \\
Receiving nodes $K_{f}$ & 4,8 \\
Path loss exponent $\alpha$ & 4 \\
SINR threshold $\gamma_{c, \text { thre }}$ & $-30 \mathrm{~dB}$ \\
SINR threshold $\gamma_{f, \text { thre }}$ & $-20,-30 \mathrm{dBm}$ \\
Distance between BS and UE $D_{i}$ & $500 \mathrm{~m}$ \\
Distance between transmitting node and receiving node $D_{k}$ & $100 \mathrm{~m}$ \\
MU-MIMO scheme in cellular cell & ZF-based \\
MU-MIMO scheme in ad hoc networks & Codebook-based \\
\hline
\end{tabular}

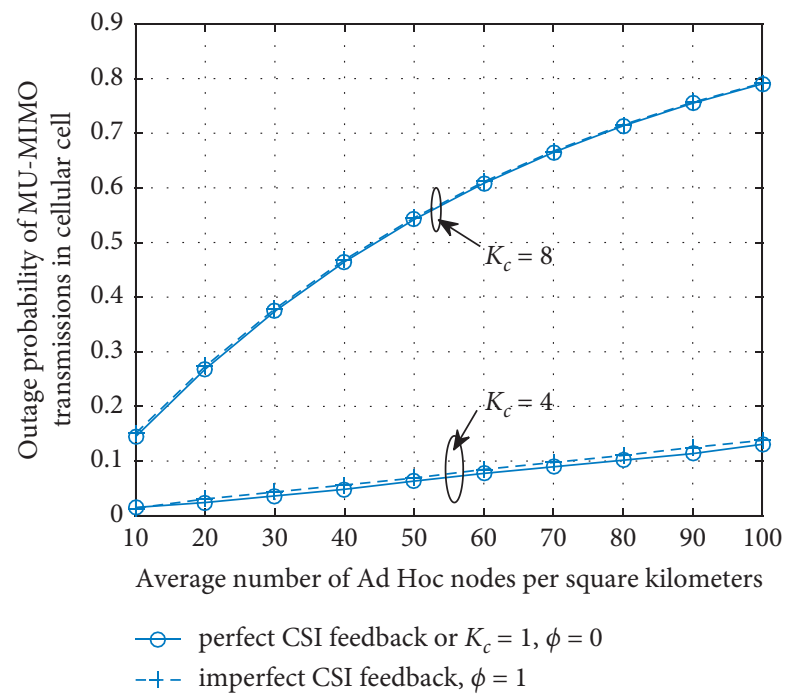

FIGURE 2: Outage probability of MU-MIMO transmissions in cellular cell vs. average number of ad hoc nodes.

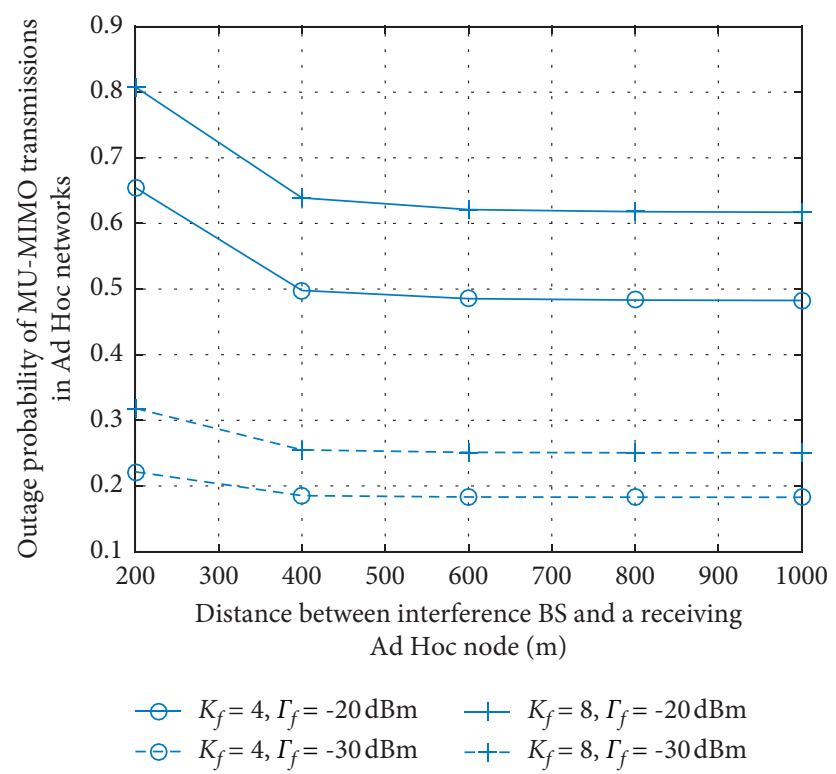

FIgURE 3: Outage probability in ad hoc networks (downlink interval) vs. distance between cellular BS and a receiving node. 


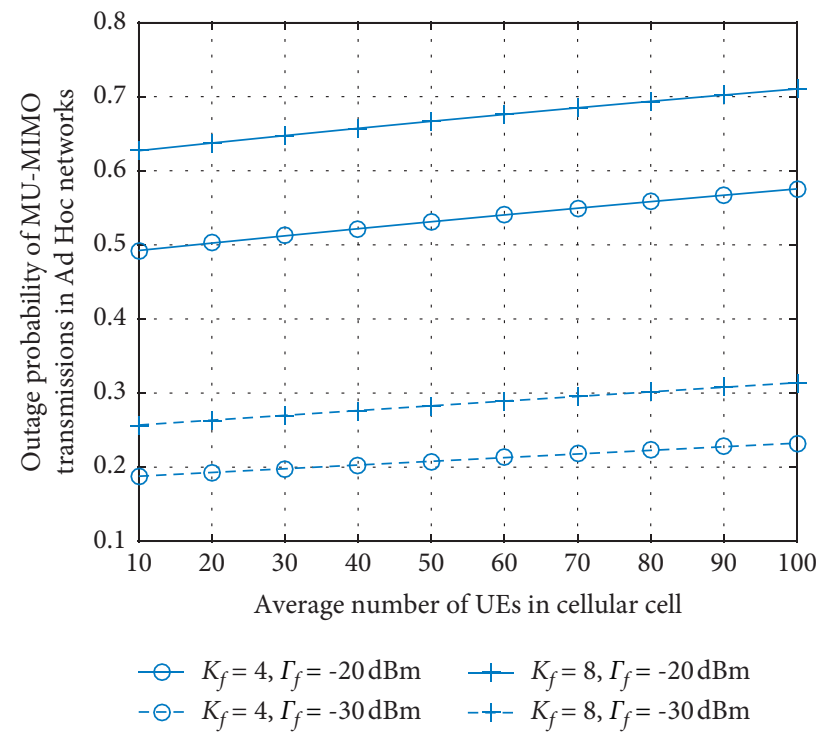

FIGURE 4: Outage probability in ad hoc networks (uplink interval) vs. average number of UE.

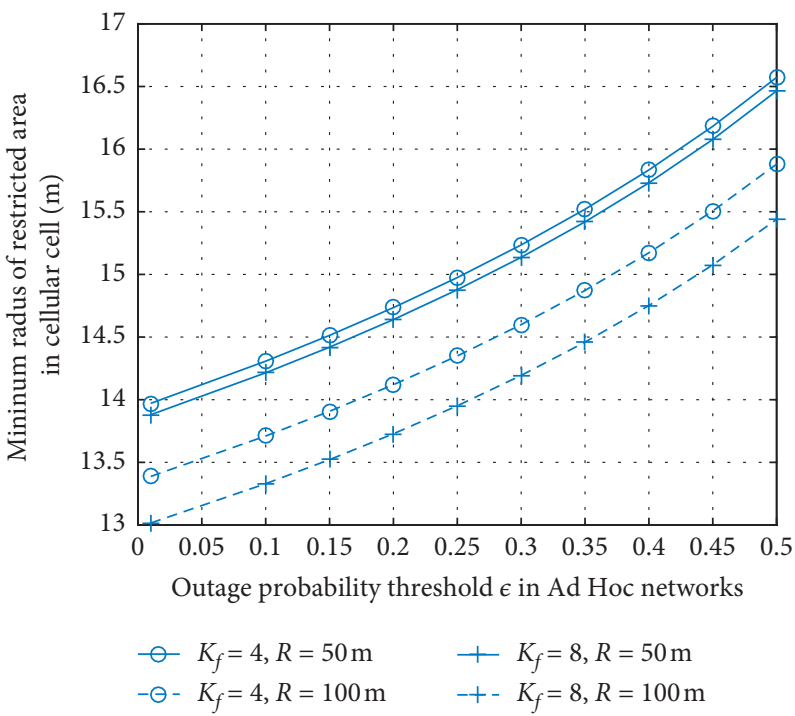

FIgURE 5: Minimum radius of restricted area in cellular cell vs. outage probability threshold in ad hoc networks.

It is obvious that there is a circle area with the BS as center and $D_{f, c}^{\min }$ as minimum radius. In the restricted area, the instantaneous outage probability of MU-MIMO transmissions in ad hoc networks does not exceed the threshold $\varepsilon$.

\section{Numerical Results}

In this section, we report the numerical results of computer simulations. In the HetNet, the coverage of cellular BS is assumed to be a circle area with $1 \mathrm{~km}$ radius. The UEs are located in the coverage area randomly. The simulation configurations are listed in Table 1.

Figure 2 shows the outage probabilities of MU-MIMO transmissions in cellular cell. With the increase of the intensity of ad hoc nodes, a UE experiences more severe crosstier interference signals. On the other hand, the increasing receiving UEs reduce the power of desired signal but may enhance the power of inter-beam interference signal. As a result, the outage probabilities of downlink MU-MIMO transmission in cellular cell increase with the intensity of the ad hoc nodes and the number of receiving UEs. Furthermore, because of the imperfect CSI feedback, the beams are prone to interfere with each other. The receiving UE will receive the inter-beam interference signal from other beams. Hence, the outage probabilities in case of imperfect CSI feedback are greater than those in case of perfect CSI feedback.

The outage probabilities of MU-MIMO transmissions in ad hoc networks in case of downlink interval and uplink interval in cellular cell are given in Figures 3 and 4, respectively. It is obvious that the number of receiving nodes and the SINR threshold have a significant effect on outage 
probability. As described in Figure 2, the increase of the receiving nodes reduces the power of desired signal but enhances the power of inter-beam interference signal. Hence, the outage probability increases with the number of receiving nodes. On the other hand, the decrease of SINR threshold reduces the probability that the received SINR does not exceed the SINR threshold. Thus, the outage probability decreases accordingly.

As shown in Figure 3, when the distance between the cellular BS and a receiving node increases, the power of crosstier interference signal the receiving node suffered is reduced. Hence, the outage probability decreases. However, if the distance exceeds a threshold, for instance $500 \mathrm{~m}$, the effect of cross-tier interference signal is not significant. The outage probability decreases with the distance slowly. In Figure 4, the growth of UEs causes more severe cross-tier interference in case uplink interval in cellular cell. As a result, the outage probability increases with the number of UEs.

Note that there is a time gap between beam selection and data transmission in MU-MIMO transmission. A receiving node may perform SINR measurement and data transmission in different intervals of cellular cell. Since the cross-tier interference is different in downlink interval and uplink interval, the instantaneous SINR in data transmission may be different from the measured SINR. From Figures 3 and 4, the instantaneous outage probability may be different from the expected outage probability.

Figure 5 shows the relation between the radius of region area in cellular cell and the outage probability threshold. When a stricter threshold is imposed on the outage probability, i.e., the threshold decreases, the outage probability of more receiving ad hoc nodes could satisfy the threshold. Taking the distribution of ad hoc nodes into account, the nodes that are close to the cellular BS and could not meet the threshold are reduced. Hence, the restricted area in cellular cell becomes smaller. Furthermore, the decrease of the minimum distance between a pair of nodes enhances the power of desired signal but reduces outage probability. Consequently, the restricted area becomes smaller when the outage probability threshold is satisfied.

\section{Conclusions}

This paper investigates per-tier outage probability of MUMIMO transmissions in HetNets with joint interference constraint. In the tier of cellular networks, a ZF-based precoding MU-MIMO scheme is employed. In the presence of cross-tier interference and unpredicted interbeam interference, the closed-form expression of outage probability of downlink MU-MIMO transmissions is derived. Considering the capacity of ad hoc nodes, a maxSINR scheduler and codebook-based MU-MIMO transmission are applied. With the constraint of joint internode interference, inter-beam interference, and downlink/uplink cross-tier interference, the closed-form expression of outage probability in ad hoc networks is obtained. Furthermore, considering the QoS requirement of ad hoc networks in case of downlink interval, the restricted area in cellular cell is explored.
As a future work, the performance optimization of MUMIMO transmissions using different interference management schemes is worthy of in-depth study.

\section{Data Availability}

The data used to support the findings of this study are available from the corresponding author upon request.

\section{Conflicts of Interest}

The authors declare that they have no conflicts of interest.

\section{References}

[1] P. Phaswana and M. Velempini, "Paper spectrum-aware transitive multicast on demand distance vector routing for military cognitive radio ad hoc networks," in Proceedings of the 2019 IEEE AFRICON, Accra, Ghana, September 2019.

[2] J. Liu and N. Kato, "A Markovian analysis for explicit probabilistic stopping-based information propagation in postdisaster ad hoc mobile networks," IEEE Transactions on Wireless Communications, vol. 15, no. 1, pp. 81-90, 2016.

[3] D. L. Guidoni, G. Maia, F. S. H. Souza, L. A. Villas, and A. A. F. Loureiro, "Vehicular traffic management based on traffic engineering for vehicular ad hoc networks," IEEE Access, vol. 8, pp. 45167-45183, 2020.

[4] Y. Zhong, T. Q. S. Quek, and X. Ge, "Heterogeneous cellular networks with spatio-temporal traffic: delay analysis and scheduling," IEEE Journal on Selected Areas in Communications, vol. 35, no. 6, pp. 1373-1386, 2017.

[5] J. Dai, W. Lv, and F. Ye, "Spectrum allocation scheme based on Stackelberg game in two-tier HetNet," in Proceedings of the IEEE 13th International Conference Signal Process, pp. 1270-1274, Chengdu, China, November 2016.

[6] H. B. Duk Kyung Kim and D. K. Kim, "Power control of femtocells based on max-min fairness in heterogeneous networks," IEEE Communications Letters, vol. 17, no. 7, pp. 1372-1375, 2013.

[7] K. Song, B. Ji, Y. Huang, M. Xiao, and L. Yang, "Performance analysis of heterogeneous networks with interference cancellation," IEEE Transactions on Vehicular Technology, vol. 66, no. 8, pp. 6969-6981, 2017.

[8] W. Zhang and S. Jiang, "Effect of node mobility on MUMIMO transmissions in mobile ad hoc networks," Wireless Communications and Mobile Computing, vol. 2021, Article ID 9954940, 9 pages, 2021.

[9] S. Lee, S. Kim, Y. Park, S. Choi, and D. Hong, "Effect of unpredictable interference on MU-MIMO systems in HetNet," IEEE Access, vol. 6, pp. 28870-28876, 2018.

[10] H. Kusumoto, H. Okada, K. Kobayashi, and M. Katayama, "Performance comparison between single-user MIMO and multi-user MIMO in wireless mesh networks," in Proceedings of the 2012 15th International Symposium on Wireless Personal Multimedia Communications, Taipei, Taiwan, September 2012.

[11] T. Erpek, Y. E. Sagduyu, Y. Shi, and S. Ponnaluri, "Rate optimization with distributed network coordination of multiuser MIMO communications," in Proceedings of the 2018 IEEE 88th Vehicular Technology Conference (VTC-Fall), Chicago, IL, USA, August 2018.

[12] L. Liu, R. Chen, S. Geirhofer, K. Sayana, Z. Shi, and Y. Zhou, "Downlink MIMO in LTE-advanced: SU-mimo 
vs. MU-MIMO," IEEE Communications Magazine, vol. 50, no. 2, pp. 140-147, 2012.

[13] R. V. Nee, "Breaking the gigabit-per-second barrier with 802.11ac," IEEE Wireless Communications, vol. 18, no. 2, p. 4, 2011.

[14] F. Kettlun, F. Rosas, and C. Oberli, "A low-complexity channel training method for efficient SVD beamforming over MIMO channels," EURASIP Journal on Wireless Communications and Networking, vol. 2021, no. 1, pp. 1-22, 2021.

[15] R. Yao, H. Nan, J. Xu, and G. Li, "Optimal BD-ZF precoder for multi-user MIMO downlink transmission," Electronics Letters, vol. 51, no. 14, pp. 1121-1123, 2015.

[16] K. M. Rege, K. Balachandran, J. H. Kang, and K. Karakayali, "Interference mitigation in heterogeneous networks with simple dirty paper coding," Wireless Networks, vol. 26, no. 4, pp. 2755-2767, 2020.

[17] J. L. Vicario, R. Bosisio, C. Anton-Haro, and U. Spagnolini, "Beam selection strategies for orthogonal random beamforming in sparse networks," IEEE Transactions on Wireless Communications, vol. 7, no. 9, pp. 3385-3396, 2008.

[18] J. Kingman, Poisson Processes, Oxford Univ. Press, London, UK., 1993.

[19] V. Chandrasekhar, M. Kountouris, and J. G. Andrews, "Coverage in multi-antenna two-tier networks," IEEE Transactions on Wireless Communications, vol. 8, no. 10, pp. 5314-5327, 2009.

[20] M. Haenggi, J. G. Andrews, F. Baccelli, O. Dousse, and M. Franceschetti, "Stochastic geometry and random graphs for the analysis and design of wireless networks," IEEE Journal on Selected Areas in Communications, vol. 27, no. 7, pp. 1029-1046, 2009.

[21] B. Blaszczyszyn, P. Mühlethaler, and Y. Toor, "Stochastic analysis of ALOHA in vehicular ad-hoc networks," Annals of Telecommunications, vol. 68, no. 1-2, pp. 95-106, 2013.

[22] F. Baccelli and A. Biswas, "On scaling limits of power law shot-noise fields," Stochastic Models, vol. 31, no. 2, pp. 1-17, 2014. 